\title{
Transcriptional profiling of Impatiens walleriana genes through different stages of downy mildew infection reveals novel genes involved in disease susceptibility
}

1 Stephanie Suarez ${ }^{1,2, a}$, Zunaira Afzal Naveed ${ }^{2,3, a}$, Gul Shad Ali ${ }^{2,3^{*}}$

$2{ }^{1}$ Tropical Research and Education Center, University of Florida, Homestead, Florida 33031, USA

$3 \quad{ }^{2}$ Department of Plant Pathology, University of Florida, Gainesville, Florida 32611, USA

$4{ }^{3}$ Mid-Florida Research and Education Center, Department of Plant Pathology, University of Florida,

5 Apopka, Florida 32703, USA

6 * Correspondence:

7 Dr. Gul Shad Ali

8 gsali@ufl.edu

$9{ }^{\text {a }}$ Authors contributed equally. 


\section{Abstract}

12 Impatiens downy mildew is a highly destructive disease of Impatiens walleriana, and economically

13 important bedding ornamental crop. This disease is caused by a recently emerged pathogen

14 Plasmopara obducens. Since both the host and pathogen are relatively less studied, there are only a

15 few genomic resources available for both I. walleriana and P. obducens. In this study, we have

16 analyzed transcriptional changes in $I$. walleriana in response to $P$. obducens infection during

17 different stages of disease development. Our main goal was to identify candidate genes that may be involved in I. walleriana susceptibility to $P$. obducens. Since the genome of $I$. walleriana is not available publicly, we constructed and optimized a de novo transcriptome assembly consisting of 73,022 transcripts. Differential expression analysis based on this optimized de novo transcriptome assembly revealed 3,000 to 4,500 differentially expressed transcripts (DETs) at $0 \mathrm{hr}, 12 \mathrm{hr}, 48 \mathrm{hr}$, $120 \mathrm{hr}$, and $240 \mathrm{hr}$ time points after infection. Functional annotation of these DETs revealed that numerous plant stress responsive genes are activated and deactivated throughout the infection cycle. Genes in the calcium signaling pathways, receptor-like kinases (RLKs) including 10 disease resistance associated RLK transcripts, powdery mildew resistance genes $(M L O)$, and many other plant stress related genes were predominantly differentially expressed in I. walleriana in response to P. obducens. Analyses reported here provides molecular insights into the disease susceptibility mechanism of the Impatiens downy mildew, and lays out a strong foundation for future studies aimed at improving downy mildew resistance in I. walleriana.

\section{Introduction}

Downy mildews are notoriously destructive diseases causing devastating losses on both agronomic and ornamental crops worldwide. With reports on wild impatiens dating back to 1877 (Farr et al. 2016), the first report of impatiens downy mildew (IDM) on cultivated I. walleriana in the United States did not occur until 2004 (Lane et al. 2005). The fatal consequences of this disease on impatiens production have gained worldwide news coverage, as impatiens was previously the top annual bedding plant in the floriculture industry. Specifically, production in Florida averaged \$20 million flats annually before IDM was first reported in the state. Since its emergence, gardening impatiens has been replaced by landscape alternatives such as begonias and the IDM-resistant New Guinea impatiens (I. hawkeri). 
obducens. Recently, the first de novo assembly of $P$. obducens resulted in a 202-

43 Mb genome (Salgado-Salazar et al. 2015). Polymorphic SSR marker candidates were discovered and will hopefully serve as an exciting new source as the ongoing molecular studies of this pathogen continues. While there is much to learn about the genetics of $P$. obducens, recent research available regarding I. walleriana was published identifying disease resistance genes as well as SSRs and SNPs sites which may aid in breeding impatiens for resistance to IDM (Bhattarai et al. 2018). There is strong consumer demand for IDM-resistant I. walleriana cultivars. In addition to studying the host and its pathogen separately, understanding the plant defenses in the host-pathogen interaction during infection is critical in order to introduce disease resistance in susceptible host cultivars.

Pattern recognition receptors (PRRs) are the first layer of plant defense in the innate immune system of plants. Localized to host cell membranes, they recognize pathogen structures or molecules known as pathogen-associated molecular pattern (PAMP) and in turn, activate PAMP-Triggered Immunity (PTI). PTI is a non-specific defense mechanism allowing for recognition of both nonpathogenic and pathogen organisms, and thus combatting pathogen invasion and further colonization. A second layer of immunity, effector-triggered immunity (ETI) may be activated if a pathogen survives PTI. ETI depends on pathogen effectors, secreted in host cells by the pathogen, resulting in programmed cell death at the site of infection, or a hypersensitive response (HR) in an incompatible reaction.

HR is prompted by host resistance $(\mathrm{R})$ genes, which are divided into five classes: (i) the CNL class comprising of $\mathrm{R}$ genes encoding a protein with at least one $\mathrm{N}$-terminal coiled-coil, a nucleotide binding site and a leucine-rich repeat (CC-NB-LRR), (ii) TNL class containing a Toll-interleukin receptor-like domain, a nucleotide binding site and a leucine-rich repeat (TIR-NB-LRR), (iii) RLP class which is a receptor-like protein containing receptor serine-threonine kinase-like domain and an extracellular leucine-rich repeat (ser/thr-LRR), (iv) RLK which are receptor-like kinase containing an extracellular leucine-rich repeat in addition to the kinase domain (Kin-LRR), (v) 'Others' class includes any other genes described as using other molecular mechanisms to confer resistance (Sanseverino et al. 2010). Bhatarrai et al. found most of their candidate genes showing high similarity levels to the NB-LRR or LRR gene families and identified two genes as TNL or CNL encoding proteins. Studying potential R genes involved in defense against IDM is paramount in order for breeding IDM-resistant impatiens in the future.

Our research focused on the host side in a compatible reaction between a susceptible cultivar of $I$. walleriana and $P$. obducens during the infection cycle. The main focus was to identify candidate genes that may be involved in impatiens susceptibility to $P$. obducens. These results could provide 
insights and lay the groundwork for future studies in understanding both host-pathogen interaction in IDM. In this study, we present findings from the first analysis of differential gene expression during the infection of $I$. walleriana by causal agent $P$. obducens.

\section{Materials and Methods}

\section{P. obducens Inoculation and Sample Collection}

Impatiens walleriana cv. 'Super Elfin White' plants were grown in growth chambers maintained at $24^{\circ} \mathrm{C}$ with a $12 \mathrm{hr}$ light/dark photoperiod. The $P$. obducens isolate was maintained on impatiens plants in the growth chamber. Fully expanded leaves of eight impatiens plants were inoculated by pipetting $50 \mu \mathrm{L}$ sporangial suspension (1x108 sporangia/mL) of $P$. obducens on the abaxial leaf surface. Infection was verified under microscopic observations by staining leaves with tryphan-blue at each time point. Leaves were collected at five different time points $(0 \mathrm{hr}, 12 \mathrm{hr}, 48 \mathrm{hr}, 120 \mathrm{hr}$, and $240 \mathrm{hr}$ ) and flash frozen in liquid nitrogen and stored at $-80^{\circ} \mathrm{C}$ until use. Each treatment (time point) consisted of two biological replicates, resulting in a total of 10 leaf tissue RNA samples. Plasmopara obducens sporangia for pathogen baseline samples were carefully scraped off the abaxial surface of impatiens leaves showing sporulation and were also flash frozen and stored before RNA isolation.

\section{Library Preparation and Sequencing}

Total RNA was isolated from $P$. obducens sporangia and inoculated impatiens leaves using the RNeasy Plant Mini Kit (Qiagen, Germantown, MD) according to manufacturer's protocols. RNA concentration and quality were determined using Bioanalyzer 2100 (Agilent Technologies, Santa Clara, CA). Library sample preparation was done using a stranded mRNA-seq kit (KAPA Biosystems, Inc., Wilmington, MA) and sequencing was performed using an Illumina HiSeq 2500 v4 platform (Illumina, Inc., San Diego, CA) at the Center for Genomic and Computational Biology (GCB) at Duke University. Each library was sequenced using the pair-end ( 2 x 125bp) protocol. A total of 24 libraries were made (Table 1).

\section{RNA-Seq Analysis}

Raw reads were imported into CLC Genomics Workbench R9 (Qiagen) and assessed for quality. Sequencing adaptors and low-quality reads were trimmed by $15 \mathrm{bp}$ and $5 \mathrm{bp}$ from the 5' and 3' ends, respectively. A trimming quality score of 0.005 was used. Cleaned high quality reads from samples were assembled de novo using the default parameters in the CLC Genomics Workbench 9.5.3. 
105 Because of the complexity of transcriptome data, a word size 25 and bubble size 1000 were used for

106 de novo assembly.

107 Assembly Optimization and Functional Annotation

108 Contigs were subsequently fed to the program cd-hi-est for removal of any redundancy in the

109 sequence, using a sequence identity threshold of 0.95 (Mu $\square$ ller et al. 2017). After cd-hit clustering

110 analysis, selected contigs were subjected to comprehensive functional annotation using Blast2GO 5

111 PRO (Conesa et al. 2005). CloudBLAST tool was used to run BLASTX on transcripts against the

112 non-redundant plant genome NCBI database using an error cutoff value of 1 x10-5. Gene ontology

113 (GO) mapping of the transcriptome was conducted following annotation of transcripts in order to

114 produce a GO distribution.

\section{Differential Expression Analysis}

116 Differentially expressed transcripts (DETs) were determined by using the R package DESeq2 using

117 default parameters. Differential expression analysis was done using the transcript counts table

118 generated in CLC genomics. The DETs were filtered at a threshold of $\log 2$ fold $>|2|$ and padj $<0.001$.

119 A Venn diagram analysis on DETs at all timepoints was done using the online tool provided by VIB

120 and Ghent University (http://bioinformatics.psb.ugent.be/webtools/Venn/). Finally, functions of the

121 DETs, specifically in common plant pathways, were determined using MapMan software.

\section{Results}

\section{RNA-Seq and de novo Transcriptome Assembly}

124 In total, 689,038,886 reads were generated from all 24 samples (libraries). After quality assessment 125 and trimming, a total of 528,964,774 clean reads were used to construct a de novo assembly in the

126 CLC Genomics workbench which resulted in 91,164 contigs with an N50 value of 1650 bp (50\% of 127 transcripts greater than or equal to 1650bp long) (Table 1). These contigs were fed into the cd-hit-est 128 cluster analysis based on similarity, producing 89,987 contigs. Filtering through BLASTx to remove $129 P$. obducens contigs resulted in 73,022 contigs. This optimized assembly was used as a reference and 130 reads from all the samples were mapped back to this optimized pooled reference assembly and a read 131 count table was generated. Differential expression analysis was done on this table using DEseq2.

132 Only those transcripts were considered as differentially expressed genes that have $\log 2$ fold $>|2|$ and 133 padj < 0.001. According to this criterion, the number of DETs between infected and control samples 134 ranged from around 3000 to 4500 among infected vs. control samples at all six time points with the 
135 highest number of DETs (4549) at $0 \mathrm{hr}$ and the lowest at $120 \mathrm{hr}$. The number of upregulated genes

136 was more than number of downregulated genes at all time points with the most upregulation

137 happening at $240 \mathrm{hr}$ (2672). Moreover, venn diagram analysis showed that the highest number of

138 DETs (502) unique to timepoint were expressed at $240 \mathrm{hr}$ (Figure 1).

\section{Sequence Annotation}

140 Functional annotation of differentially expressed genes was conducted using Blast2GO software.

141 Species distribution showed little homology with other plant species. There is a lack of impatiens

142 genes available within the NCBI database, therefore the species available with the largest number of

143 BLAST hits was Vitis vinifera (Figure 2). Gene ontology (GO) terms were assigned to DEGS among

144 all three GO categories i.e. biological process (BP), molecular function (MF) and cellular component

145 (CC). Most BP hits were placed in the categories of catalytic and binding and transport activity. The

146 DETs in the MF category were mostly placed within metabolic and cellular processes, response to

147 stimulus and biological regulation. In CC, most DETs fell under the membrane category (Figure 3).

148 We generated MapMan figures at each time point in order to provide a visual representation of genes

149 involved in common plant pathways (Thimm et al. 2004; Usadel et al. 2009). MapMan annotation is

150 only available for few plant species, therefore we used the Arabidopsis homologs for our de novo

151 constructed impatiens transcripts.

152 DEGs from all six time points were mapped on the biotic stress pathways. Genes involved in

153 proteolysis were observed to be mainly upregulated across all time points (Figures 4). General

154 signaling genes were about equally downregulated as they were upregulated. The WRKY

155 transcription factors (TFs) were mostly upregulated.

\section{Genes Involved in Plant Defense}

157 Many of already reported plant defense-related genes were found to be differentially expressed at all

158 time points. For example, multiple calcium signaling genes were found differentially expressed at all

159 time points. Most of the calcium binding genes were found to be upregulated, whereas calcium

160 sensing receptors were found to be downregulated across all time points (Table 2).

161 Many of the receptor-like kinases (RLKs) were found to be differentially expressed across all time 162 points, including eleven leaf rust 10 disease resistance receptor-like protein kinase transcripts, fifteen 
163 G-type lectin S-receptor-like serine/threonine-protein kinase transcripts and five wall-associated

164 receptor kinases. These were found to be mostly upregulated.

165 We found six different homologs of MLO genes differentially expressed across all time points. Three 166 of these transcripts were upregulated and three were downregulated (Figure 5). Apart from the

167 aforementioned genes, we found many other core plant defense genes differentially expressed in $I$.

168 walleriana in response to infection by $P$. obducens at all stages of the infection cycle.

\section{Discussion}

170 RNA-Seq is an excellent technique to reveal changes in a plant's gene expression in response to

171 pathogens, even when whole genome information is unavailable. A recent study was published which

172 is the first comparative transcriptome analysis of both a resistant and susceptible impatiens cultivar in

173 order to identify candidate genes for IDM resistance (Bhattarai et al. 2018). This study was

174 conducted on non-inoculated, uninfected impatiens leaves. To further host-pathogen interaction, we

175 analyzed transcriptional changes in an inoculated, susceptible cultivar of impatiens at 6 timepoints.

176 At 10 days $(240 \mathrm{hr})$ post-inoculation, sporulation of $P$. obducens can be observed on the abaxial leaf

177 surface of susceptible, infected impatiens plants. Plasmopara obducens is an obligate biotroph,

178 therefore we wanted to track transcriptional changes throughout the entirety of its infection cycle $(0$

179 to $240 \mathrm{hr}$ ). To our knowledge, this is the first study of the host-pathogen interaction between $I$.

$180 \quad$ walleriana and P. obducens available.

181 In 2018, Ball Horticultural Company and KeyGene announced the completion of the entire genome

182 sequencing and assembly of I. walleriana. Because it is not currently available to the public, our

183 study relied on the use of de novo transcriptome assembly of I. walleriana. After assembly,

184 optimization was done in order to preserve biologically meaningful transcripts for further differential

185 expression analysis. Differential gene expression among infected and control samples were observed

186 immediately after inoculation. In order to track transcriptional changes during the first contact

187 between plant and pathogen, we collected samples for the first time point immediately following

188 inoculation, designated as 0hr. Surprisingly, we observed the highest number of differentially

189 expressed genes at $0 \mathrm{hr}$ than at any other timepoint, except $240 \mathrm{hr}$.

190 Plant pathogens contain specific structures or molecules known as pathogen associated molecular

191 patterns (PAMP), which are recognized by PRRs to active plant defense. This is known as PAMP-

192 Triggered Immunity (PTI). Once activated, PTI results in basal defense responses to pathogen 
193 invasion (Dodds and Rathjen 2010). The present study showed the differential expression of all kinds

194 of PRRs in susceptible impatiens infected with P. obducens at Ohr. Plants carry two types of PRRs:

195 receptor- like kinases (RLKs) and receptor-like proteins (RLPs) (Tang et al. 2017). Wall- associated

196 RLKs have been reported as the positive regulators of plant defense against fungi (Delteil et al.

197 2016). In our study, we found five wall-associated receptor-like kinases to be upregulated.

198 Another type of RLK, G-type lectin S-receptor-like serine/threonine-protein kinase, was also found 199 to be upregulated across all timepoints, including $0 \mathrm{hr}$. These lectin receptor kinases are a class of 200 RLKs divided into three subclasses (C-type, G- type and L-type). The G-type kinase that we have 201 found upregulated in this study has yet to be reported in oomycete-plant interactions. Further studies

202 may be able to confirm if G-type receptor kinases play a role in plant defense against oomycetes. We 203 also found eleven leaf rust 10 disease resistance receptor-like protein kinase isoforms to be 204 upregulated as well. This is an indication that the impatiens plant recognizes infection by $P$. obducens immediately upon inoculation, prompting plant defenses. Membrane-associated PRRs have previously been reported to be involved in initial plant-pathogen interactions, but we observed the continuation of upregulation of these RLKs throughout the entire infection cycle of $P$. obducens in $I$. walleriana.

After recognition by PRRs, calcium ion $(\mathrm{Ca} 2+)$ concentrations are considered to be the next event in plant responses to any environmental cues (Ranty et al. 2016). Many calcium signaling genes in this study were found to be differentially expressed starting at initial contact between $P$. obducens and $I$. walleriana. Our study focused on a compatible interaction between a susceptible cultivar of $I$.

213 walleriana and P. obducens. Many transcriptome studies have revealed there is involvement of Ca2+

214 signaling genes in compatible host-pathogen interactions (Aldon et al. 2018; Naveed and Ali 2018).

215 MLO proteins were initially identified as common powdery mildew susceptibility genes, but recent

216 studies have found them to be involved in plant-oomycete interactions as well (Zhu et al. 2017;

217 Naveed and Ali 2018). We found six homologs of MLO genes to be differentially expressed in $I$.

218 walleriana, three of which were highly upregulated. Induction of MLO genes by P. obducens in I.

219 walleriana could be speculated as another example of MLO genes being involved in host

220 susceptibility to pathogens other than powdery mildew, in this case, an oomycete.

221 In addition to the aforementioned genes, we found many other core plant defense-related genes such 222 as pathogenicity-related genes, resistance genes, and biotic stress-related transcription factors to be 
223 differentially expressed throughout the infection cycle of IDM. This study revealed the presence of

224 transcriptional changes in common physiological and core plant defense-related pathways in $I$.

225 walleriana during infection by $P$. obducens. Many candidate genes have been identified in this study,

\section{References}

Aldon, D., Mbengue, M., Mazars, C., and Galaud, J.-P. 2018. Calcium signaling in plant biotic interactions. Int. J. Mol. Sci. 19: 665.

230 Bhattarai, K., Wang, W., Cao, Z. and Deng, Z. 2018. Comparative analysis of impatiens leaf transcriptomes reveal candidate genes for resistance to downy mildew caused by Plasmopara obducens. Int. J. Mol. Sci. 19: 2057.

Delteil, A., Gobbato, E., Cayrol, B., Estevan, J., Michel-Romiti, C., Dievart, A., Kroj, T., and Morel, 234 J. B. 2016. Several wall-associated kinases participate positively and negatively in basal defense against rice blast fungus. BMC Plant Biology. 16:17.

236 Dodds, P. N., and Rathjen, J. P. 2010. Plant immunity: towards an integrated view of plant-pathogen

237 interactions. Nature Reviews Genetics. 11: 539-548.

238 Farr, D. F., and Rossman, A. Y. 2016. Fungal Databases, Systematic Mycology and Microbiology

239 Laboratory, ARS, USDA.

240 Lane, C. R., Beales, P. A., O’Neill, T. M., McPherson, G. M., Finlay, A. R., David, J.,

241 Constantinescu, O., and Henricot, B. 2005. First report of Impatiens downy mildew (Plasmopara

242 obducens) in the UK. Plant Pathol. 54:243.

243 Naveed, Z. A., and Ali, G. S. 2018. Comparative transcriptome analysis between a resistant and a

244 susceptible wild tomato accession in response to Phytophthora parasitica. Int. J. Mol. Sci. 19: 3735.

245 Salgado-Salazar, C., Rivera, Y., Veltri, D., and Crouch, J. A. 2015. Polymorphic SSR markers for

246 Plamopara obducens (Peronosporaceae), the newly emergent downy mildew pathogen of Impatiens

247 (Balsaminaceae). Appl. Plant. Sci. 3: 1500073.

248 Sanseverino, W., Roma, G., De Simone, M., Faino, L., Melito, S., Stupka, E., Frusciante, L., and 249 Ercolano, M.R. 2010. Nucleic Acids Res. 38: D814-D821.

250 Tang, D., Wang, G., and Zhou, J. 2017. Receptor kinases in plant-pathogen interactions: more than 251 pattern recognition. Plant Cell. 29: 618-637.

252 Thimm, O., Blasing, O., Gibon, Y., Nagel, A., Meyer, S., Kruer, P., Selbig, J., Muller, L. A., Rhee, S. 253 Y., and Stitt, M. 2004. Mapman: a user driven tool to display genomics data sets onto diagrams of metabolic pathways and other biological processes. The Plant Journal. 37: 914-939. 
255

256

257

258

259

260

261

262

263

264

265

266

267
Zhu, Y., Shao, J., Zhou, Z., and Davis, R.E. 2017. Comparative transcriptome analysis reveals a preformed defense system in apple root of a resistant genotype of G.935 in the absence of pathogen. Int. J. Plant Genomics. 2017: 895076.

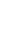

Table 1: Statistical summary of RNAseq data.

\begin{tabular}{|c|c|c|}
\hline Sample ID & Raw Reads (PE) & Clean Reads \\
\hline 0H_Control_R1 & $23,520,868$ & $18,287,675$ \\
\hline OH_Control_R2 & $27,793,058$ & $24,193,950$ \\
\hline 12H_Control_R1 & $24,320,452$ & $20,299,572$ \\
\hline 12H_Control_R2 & $19,672,888$ & $15,319,055$ \\
\hline 24H_Control_R1 & $24,320,452$ & $21,024,513$ \\
\hline 24H_Control_R2 & $19,672,888$ & $13,922,270$ \\
\hline 48H_Control_R1 & $24,162,182$ & $17,057,095$ \\
\hline 48H_Control_R2 & $17,226,072$ & $19,581,661$ \\
\hline 120H_Control_R1 & $20,526,634$ & $23,639,470$ \\
\hline 120H_Control_R2 & $23,321,216$ & $18,259,179$ \\
\hline
\end{tabular}




\begin{tabular}{|c|c|c|}
\hline 240H_Control_R1 & $27,654,810$ & $14,085,034$ \\
\hline 240H_Control_R2 & $21,765,004$ & $21,712,471$ \\
\hline OH_Infected_R1 & $37,971,712$ & $29,005,241$ \\
\hline OH_Infected_R2 & $37,778,302$ & $29,248,606$ \\
\hline 12H_Infected_R1 & $33,006,920$ & $24,968,942$ \\
\hline 12H_Infected_R2 & $31,223,426$ & $24,440,783$ \\
\hline 24H_Infected_R1 & $34,379,442$ & $26,500,567$ \\
\hline 24H_Infected_R2 & $36,662,544$ & $27,860,148$ \\
\hline 48H_Infected_R1 & $31,490,402$ & $23,140,744$ \\
\hline 48H_Infected_R2 & $33,238,084$ & $24,795,526$ \\
\hline 120H_Infected_R1 & $37,946,602$ & $29,078,825$ \\
\hline 120H_Infected_R2 & $34,596,692$ & $25,346,127$ \\
\hline 240H_Infected_R1 & $33,746,888$ & $23,867,301$ \\
\hline 240H_Infected_R2 & $33,041,348$ & $24,552,405$ \\
\hline Total & & $528,964,774$ \\
\hline
\end{tabular}

269 Table 2: Pairwise comparisons between infected vs. control samples at all six timepoints.

\begin{tabular}{lcccc}
$\begin{array}{l}\text { Comparison } \\
\text { (infected vs. control) }\end{array}$ & Total DETs & Upregulated DETs & Downregulated DETs & Unique to timepoint DETs \\
\hline 0 hour & 4549 & 2471 & 2068 & 298 \\
12 hour & 4098 & 2132 & 1966 & 140 \\
24 hour & 4360 & 2236 & 2133 & 201
\end{tabular}




$\begin{array}{lllll}48 \text { hour } & 3620 & 2006 & 1613 & 112 \\ 120 \text { hour } & 3607 & 2066 & 1541 & 97 \\ 240 \text { hour } & 4491 & 2672 & 1819 & 502\end{array}$

277 Figure 1: Venn diagram of pair-wise comparisons of DETs unique to timepoints.

278 Figure 2: Species distribution showing maximum homology with plant species.

279 Figure 3: GO sequence distribution chart separated by biological process (BP), molecular function 280 (MF) and cellular component (CC).

281 Figure 4: Visualization of differentially expressed genes involved in biotic stress pathways in 282 response to $P$. obducens (A) at Ohpi (hours post-inoculation), (B) at $12 \mathrm{hpi}$, (C) at 24hpi, (D) at 48hpi, 283 (E ) at $120 \mathrm{hpi}$ and (F) at 240hpi. Color gradient represents $\log 2$ fold ratios with red representing 284 upregulation and green representing downregulation in treatments over mock roots. Each box 285 represents one transcript.

286 Figure 5: Heat map showing expression profiles of MLO genes. 
bioRxiv preprint doi: https://doi.org/10.1101/622480; this version posted May 13, 2019. The copyright holder for this preprint (which was not certified by peer review) is the author/funder, who has granted bioRxiv a license to display the preprint in perpetuity. It is made available under aCC-BY-NC-ND 4.0 International license. 
$48 \mathrm{hr}$

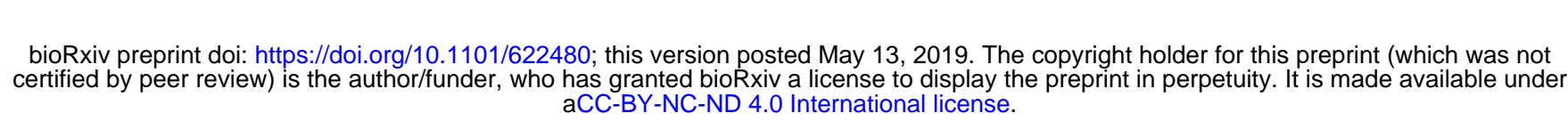

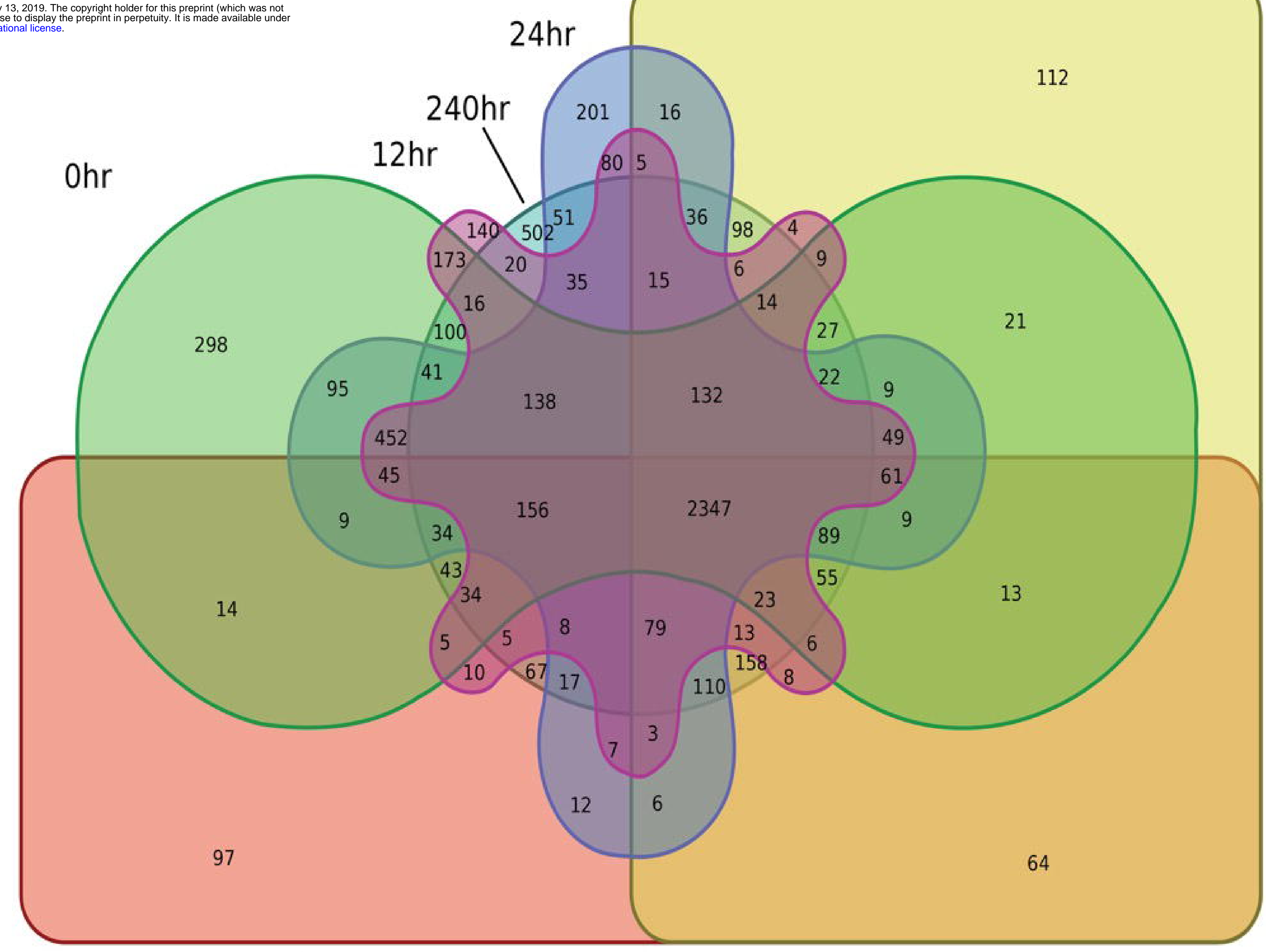

$120 \mathrm{hr}$ 
Species Distribution [impatiense_dets]

\#BLAST Hits

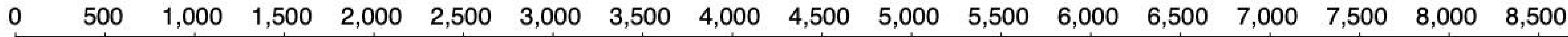

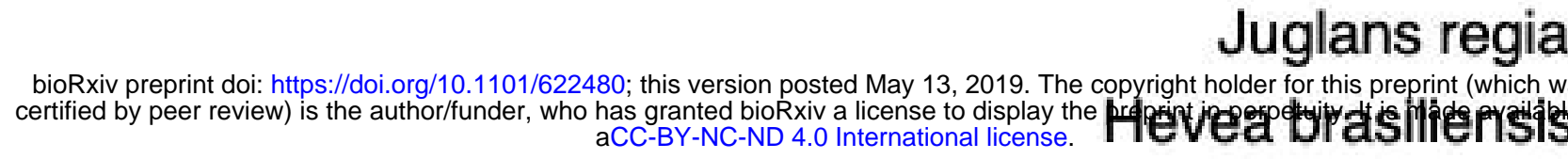

Nicotiana attenut

Nicotiana tomentosiformis

Coffea canephora

Nicotiana sylvestris

Manihot esculenta

Citrus sinensis

Nelumbo nucifera

Jatropha curcas

\% Daucus carota subsp. sativus

के

Ziziphus jujuba

Ipomoea nil

Populus trichocarpa

Gossypium hirsutum

Ricinus communis

Populus euphratica

Solanum tuberosum

Prunus persica

Herrania umbratica

Erythranthe guttata

Prunus avium

Solanum pennellii

Gossypium raimondi

Solanum lycopersicum others

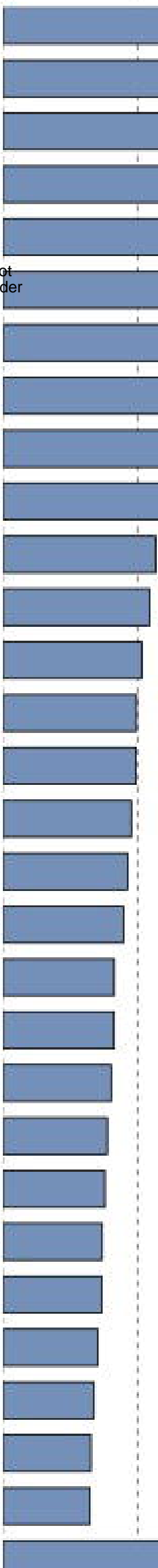




\section{GO Distribution by Level (2) - Top 20}

$\begin{array}{llllllllllllllll}100 & 200 & 300 & 400 & 500 & 600 & 700 & 800 & 900 & 1,000 & 1,100 & 1,200 & 1,300 & 1,400 & 1,500 & 1,600\end{array}$

metabolic process

ological regulation

response to stimulus

regulation of biological process

cellular compon

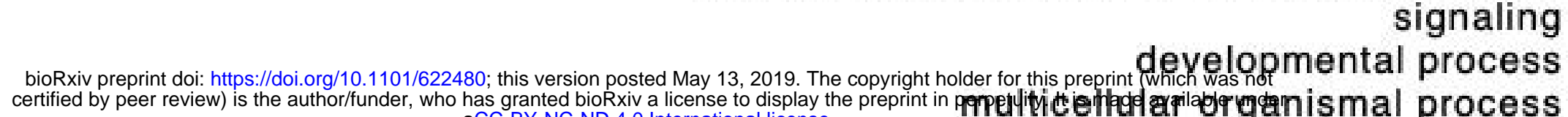

की

detoxification
multi-organism process

reproduction
rective process

egative regulation of biological process

positive regulation of biological process

immune system process
growth

rhythmic process

carbon utilization

catalytic activity

transporter activity

transcription

structural molecule activity
signal transducer activity

molecular fun

nutrient reservoir activity
molecular carrier activity

$$
\text { membrane }
$$

cell
cell part
membrane part

organelle
ganelle part

protein-containing complex
extracellular region

membrane-enclosed lumen

cell junction
symplast

extracellular region part

supramolecular complex
nucleoid
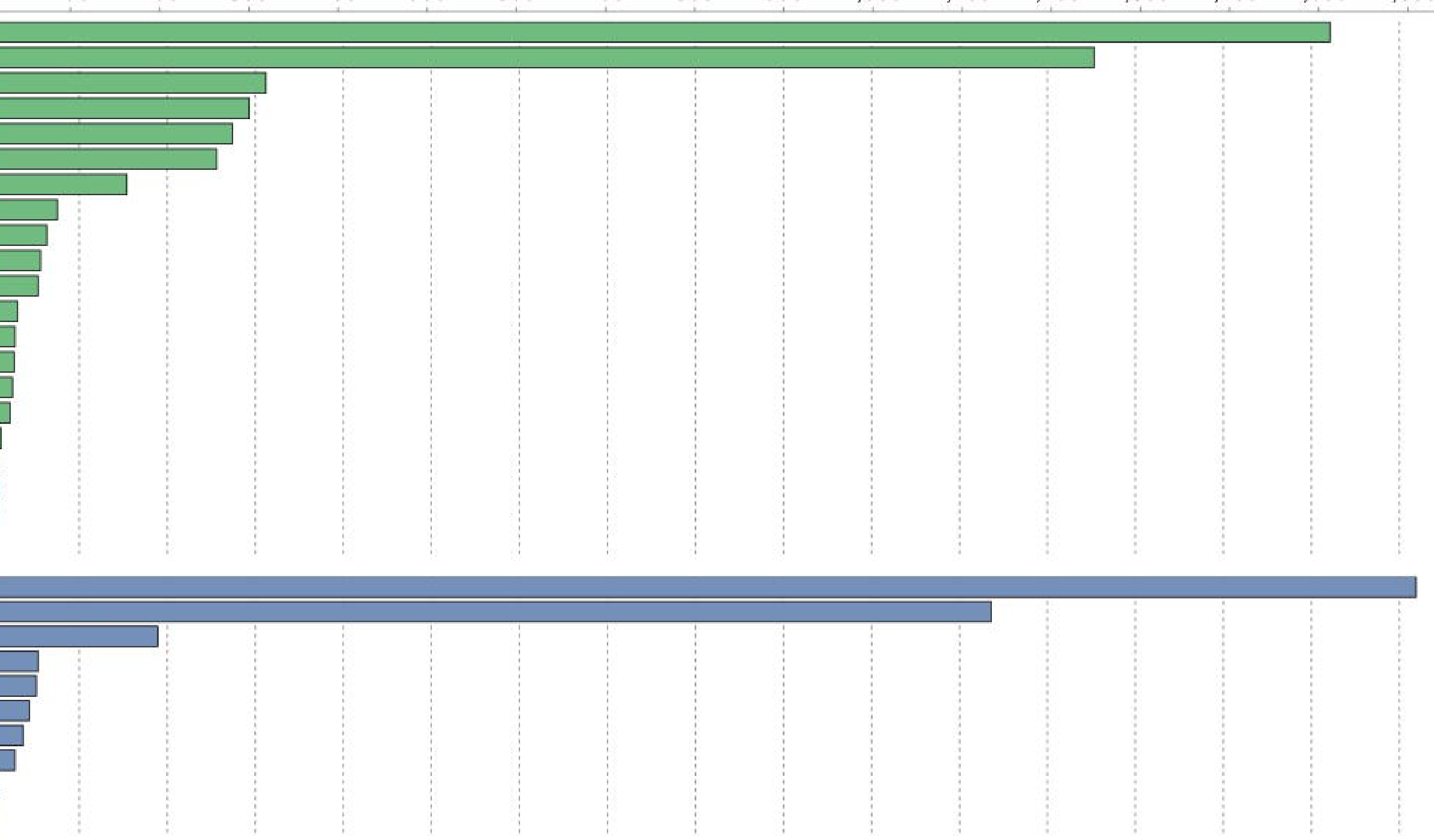

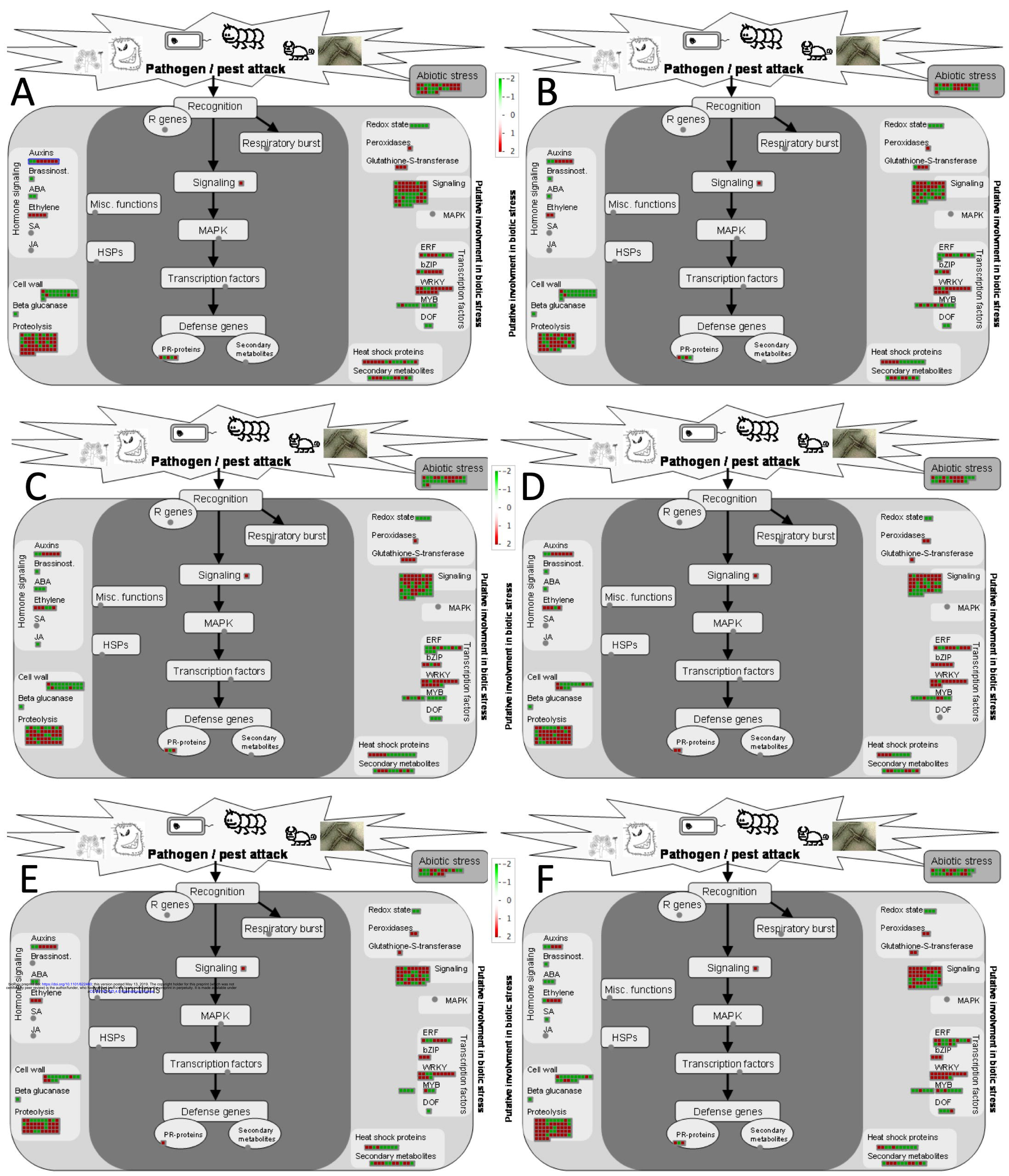
MLO-like protein 13

MLO protein

MLO-like protein 3 isoform $\mathrm{X} 4$

MLO-like protein 3

MLO-like protein 6

MLO-like protein 6 